\title{
Mobile Tools for Community Scientists
}

\author{
Maria Antonia Brovelli *, Candan Eylül Kilsedar and Francesco Frassinelli \\ Politecnicodi Milano,maria.brovelli@polimi.it, candaneylul.kilsedar@polimi.it,francesco.frassinelli@mail.polimi.it \\ * Corresponding author
}

Keywords: Mobile, Citizen Science, Community Science, Participatory Sensing

\begin{abstract}
:
While public participation in scientific achievements has a long history, the last decades have seen more attention and an impressive increase in the number of involved people. Citizen science, the term used for denoting such an attitude, is a very diverse practice, encompassing various forms, depths, and aims of collaboration between scientists and citizen researchers and a broad range of scientific disciplines. Different classifications of citizen science projects exist based on the degrees of influence and contributions of citizens. Haklay, Mazumdar, and Wardlaw (2018) distinguish the citizen
\end{abstract} science projects in three different classes:

1. Long-running citizen science, which are the traditional ones, the projects similar to those run in the past (Kobori et al., 2016; Bonney et al., 2009)

2. Citizen cyberscience, strictly connected with the use of technologies (Grey, 2009) and which can be subclassified in:

2.1. volunteer computing, where citizens offer the unused computing resources of their computers;

2.2. volunteer thinking, where citizens offer their cognitive abilities for performing tasks difficult for machines;

2.3. passive sensing, where citizens use the sensors integrated into mobile computing devices to carry out automatic sensing tasks.

3. Community science, involving a more significant commitment of citizens also in designing and planning the project activities in a more egalitarian (if not bottom-up) approach between scientists and citizen scientists (Jepson \& Ladle, 2015; Nascimento, Guimarães Pereira, \& Ghezzi, 2014; Breen, Dosemagen, Warren, \& Lippincott, 2015), which can be divided into:

3.1. participatory sensing, where citizens use the sensors integrated into mobile computing devices to carry out sensing tasks;

3.2. Do It Yourself (DIY) science, which implies participants create their scientific tools and methodology to carry out their researches;

3.3. civic science, “which is explicitly linked to community goals and questions the state of things” (Haklay et al., 2018).

The work presented here is of interest of community scientists which voluntarily offer their time for the development of scientific projects. Many software tools have been developed in order to simplify the insertion of data into structured forms and the aggregation and analysis of the obtained data. In recent years, the growing availability of feature-rich and low-cost smartphones have boosted the development of innovative solutions for data collection using portable devices. In this field, ODK (OpenDataKit) is widely known. It is an open-source suite of tools focused on simplicity of use, which includes an Android application for data collection. We used ODK for the first applications we developed.

One of the applications we developed using ODK is Via Regina (http://www.viaregina.eu/app). The application aims to support slow tourism in Via Regina, which is a road that overlooks the west coast of Lake Como in Northern Italy. Over the centuries, Via Regina has been a critical trade and pilgrim route in Europe. Moreover, from this road, a compact system of slow mobility paths departs, which span the mountainous region at the border between Italy and Switzerland. This region is rich in culture, regarding history, art, architecture, cuisine and people's lifestyle. Considering collecting data on Via Regina and the paths around it would enable to rediscover and promote its culture while enjoying the territory, an Interreg project named “The Paths of Regina” started. The application developed within this project allows collecting data in predefined types: historical and cultural, morphological, touristic, and critical. Moreover, while reporting a point of interest (POI), the application asks the name, the position (through GPS or an interactive map), a picture, and optionally a video and an audio record of it (Antonovic et al., 2015).

However, since ODK application can be used only on Android devices, we developed a cross-platform application to collect similar data for the same purpose. It is available on Android, iOS, and web (http://viaregina3.como.polimi.it/app/). The application is developed using Apache Cordova, which is a mobile application development framework that enables running the application in multiple platforms. Leaflet library is used for web mapping. The data is stored in NoSQL 
PouchDB and CouchDB database, which enables both online and offline data collection. While reporting a POI, the application asks for its type, the user's rating, a comment, and a picture of it either uploaded from device's storage or taken using the camera of the mobile device. In addition to being cross-platform, it has the advantage of displaying and enabling the query of POIs reported, compared to the ODK-based version (Brovelli, Kilsedar, \& Zamboni, 2016). Regarding citizen science, besides the citizens using these two applications, Iubilantes, a voluntary cultural organization, has been involved in the project as community scientists. Iubilantes created slow mobility paths to walk in and around Via Regina, using their experience gained through studying ancient paths while protecting and enhancing their assets since 1996.

Mobile data collection can also be used to compensate for the lack of reference data available for land cover validation. We developed the Land Cover Collector (https://github.com/kilsedar/land-cover-collector) application for this purpose, which collects data using the nomenclature of GlobeLand30. GlobeLand30 is the first global land cover map at 30-meter resolution, provided by National Geomatics Center of China, available for 2000 and 2010 (Chen et al., 2015). There are ten land cover classes in the GlobeLand30 dataset, which are: artificial surface, bare land, cultivated land, forest, grassland, permanent snow and ice, shrubland, tundra, water body, and wetland. The collected data will be used for validating GlobeLand30 (Kilsedar, Bratic, Molinari, Minghini, \& Brovelli, 2018). The data is licensed under the Open Database License (ODbL) v1.0 and can be downloaded within the application in JSON format. The application is currently available in eight languages: English, Italian, Arabic, Russian, Chinese, Portuguese, French and Spanish. The technologies used are the same as the cross-platform Via Regina application. As a result, it is available on Android, iOS, and web (https://landcover.como.polimi.it/collector/); and it supports display and query of the collected data. While reporting a POI, the application asks the land cover class of it, the user's degree of certainty on the correctness of the stated class, photos in north, east, south and west directions, and the user's comment. Three hands-on workshops were given to teach this application and various ways to validate GlobeLand30: the first on September 1, 2018 at the World Bank in Dar es Salaam, Tanzania (in conjunction with the FOSS4G 2018 conference); the second on September 3, 2018 at the Regional Centre for Mapping of Resources for Development (RCMRD) in Nairobi, Kenya; and the third on October 1, 2018 at the Delft University of Technology in Delft, Netherlands. The workshops, run by representatives of the project's principal investigators-Politecnico di Milano (Italy) and the National Geomatics Center of China (China) —were attended by a total of 100 people with a background in GIS and remote sensing. (Brovelli et al., 2018).

Nonetheless, there are no widely adopted cross-platform open-source solutions or systems for on-site surveys that address the problem of information silos: isolated databases, where the information is not adequately shared but rather remains sequestered within each system, which is an obstacle to using data mining to make productive use of data of multiple systems.

PSAB (Participatory Sensing App Builder) is a platform that provides an open-source and easy to use cross-platform solution for the creation of custom smartphone applications as well as web applications and catalog service for publishing the data and make them available to everyone. It takes advantage of established standards (like XLSForm for defining the structure of the form and DublinCore for exposing metadata) as well as less known yet effective solutions, like WQ (https://wq.io), a framework developed for building reusable software platforms for citizen science. These technologies have been merged, together with other software like Django, PyCSW, PostgreSQL, in a single solution, in order to assist the user during the entire process, from the definition of the form structure, to the creation of an ad-hoc application and the publication of the collected data, inside a flexible and open-source platform.

Users registered to PSAB are allowed to create a new application by filling a web form where they can upload their XLSForm files and submit the metadata describing the data to be collected. A new application for collecting data on the field is generated and accessible via web and Android (while iOS requires a particular setup), ready to be used online and offline. The creator of each application is also the administrator of it, which means he/she is allowed to add or ban users and modify or remove existing data. Data is automatically synchronized between all the users participating in the project.

In the presentation we will show the applications we developed, starting from the ODK-based ones and coming to the PSAB application builder, and our experience related to their usage.

\section{References}

Antonovic M., Brovelli M. A., Cannata M., Cardoso M., Kilsedar C. E., Minghini M., Zamboni G. (2015). Promoting slow tourism through FOSS4G Web Mapping: an Italian-Swiss case study. Geomatics Workbooks, 12, 99-104.

Bonney R., Ballard H., Jordan R., McCallie E., Phillips T., Shirk J., Wilderman C. C. (2009). Public Participation in Scientific Research: Defining the Field and Assessing Its Potential for Informal Science Education. Retrieved from http://www.birds.cornell.edu/citscitoolkit/plublications/CAISE-PPSR-report-2009.pdf

Breen J., Dosemagen S., Warren J., Lippincott M. (2015). Mapping Grassroots: Geodata and the structure of communityled open environmental science. ACME: An International Journal for Critical Geographies, 14(3), 849-873. Retrieved from https://www.acme-journal.org/index.php/acme/article/view/1236 
Brovelli, M. A., Minghini, M., Molinari, M. E., Kilsedar, C. E., Wu, H., Zheng, X., Chen, J., and Shu, P. (2018). Open source software and open educational material on land cover maps intercomparison and validation. The International Archives of the Photogrammetry, Remote Sensing and Spatial Information Sciences, XLII-4, 61-68. https://doi.org/10.5194/isprs-archives-XLII-4-61-2018

Brovelli M. A., Kilsedar C. E., Zamboni G. (2016). Visualization of VGI data through the new NASA Web World Wind virtual globe. The International Archives of the Photogrammetry, Remote Sensing and Spatial Information Sciences, XLIB4, 205-209. https://doi.org/10.5194/isprs-archives-XLI-B4-205-2016

Chen, J., Chen, J., Liao, A., Cao, X., Chen, L., Chen, X., He, C., Han, G., Peng, S., Lu, M., Zhang, W., Tong, X., and Mills, J. (2015). Global land cover mapping at $30 \mathrm{~m}$ resolution: A POK-based operational approach. ISPRS Journal of Photogrammetry and Remote Sensing, 103, 7-27.

Grey F. (2009, April 29). Viewpoint: The age of citizen cyberscience. CERN Courier. Retrieved from http://cerncourier.com/cws/article/cern/38718

Haklay M., Mazumdar S., Wardlaw J. (2018). Citizen Science for Observing and Understanding the Earth. Earth Observation Open Science and Innovation, 15, 69-88. https://doi.org/10.1007/978-3-319-65633-5_4

Jepson P., Ladle R. J. (2015). Nature apps: Waiting for the revolution. Ambio, 44(8), 827-832. https://doi.org/10.1007/s13280-015-0712-2

Kilsedar C. E., Bratic G., Molinari M. E., Minghini M., Brovelli M. A. (2018). Open educational resources for the validation of global high-resolution land cover maps. PeerJ Preprints. https://doi.org/10.7287/peerj.preprints.27214v1

Kobori H., Dickinson J. L., Washitani I., Sakurai R., Amano T., Komatsu N., Kitamura W., Takagawa S., Koyama K., Ogawara T., Miller-Rushing A. J. (2016). Citizen science: a new approach to advance ecology, education, and conservation. Ecological Research, 31(1), 1-19. https://doi.org/10.1007/s11284-015-1314-y

Nascimento S., Guimarães Pereira Â., Ghezzi A. (2014). From Citizen Science to Do It Yourself Science: An annotated account of an on-going movement. https://doi.org/10.2788/12246 